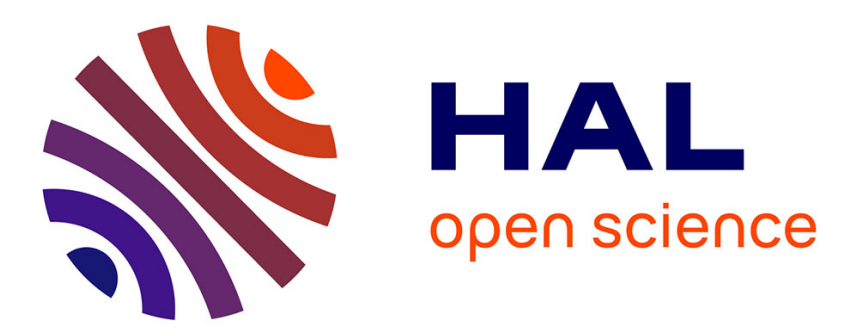

\title{
Experimental control of natural perturbations in channel flow
}

\author{
Fabien Juillet, J. Mckeon, Peter J. Schmid
}

\section{To cite this version:}

Fabien Juillet, J. Mckeon, Peter J. Schmid. Experimental control of natural perturbations in channel flow. Journal of Fluid Mechanics, 2014, 752 (august), pp.296-309. 10.1017/jfm.2014.317 . hal01057755

\section{HAL Id: hal-01057755 \\ https://hal-polytechnique.archives-ouvertes.fr/hal-01057755}

Submitted on 25 Aug 2014

HAL is a multi-disciplinary open access archive for the deposit and dissemination of scientific research documents, whether they are published or not. The documents may come from teaching and research institutions in France or abroad, or from public or private research centers.
L'archive ouverte pluridisciplinaire HAL, est destinée au dépôt et à la diffusion de documents scientifiques de niveau recherche, publiés ou non, émanant des établissements d'enseignement et de recherche français ou étrangers, des laboratoires publics ou privés. 


\title{
Experimental control of natural perturbations in channel flow
}

\author{
Fabien Juillet $^{1}$, B. J. McKeon ${ }^{2}$ and Peter J. Schmid ${ }^{3} \dagger$ \\ ${ }^{1}$ Laboratoire d'Hydrodynamique (LadHyX), CNRS-Ecole Polytechnique, 91128 Palaiseau, France \\ ${ }^{2}$ Graduate Aerospace Laboratories (GALCIT), California Institute of Technology, Pasadena, \\ CA 91125, USA \\ ${ }^{3}$ Department of Mathematics, Imperial College London, London SW7 2AZ, UK \\ (Received 16 April 2013; revised 28 March 2014; accepted 31 May 2014; \\ first published online 4 July 2014)
}

A combined approach using system identification and feed-forward control design has been applied to experimental laminar channel flow in an effort to reduce the naturally occurring disturbance level. A simple blowing/suction strategy was capable of reducing the standard deviation of the measured sensor signal by $45 \%$, which markedly exceeds previously obtained results under comparable conditions. A comparable reduction could be verified over a significant streamwise extent, implying an improvement over previous, more localized disturbance control. The technique is effective, flexible, and robust, and the obtained results encourage further explorations of experimental control of convection-dominated flows.

Key words: flow control, instability control

\section{Introduction}

Despite a great deal of progress in the development and application of flow control strategies, there are rather few experimental efforts that achieve the kind of control results that so readily can be realized in numerical simulations. Most computational studies have concentrated on the implementation of optimal and robust control laws and have validated performance measures under controlled or designed environments. Starting from a set of governing equations and empirical assumptions about ambient noise or model uncertainties, a variational approach yields control and estimation units that aim at optimally or robustly suppressing instabilities, decreasing amplification rates, reducing drag and other undesirable output, delaying transition, avoiding separation, enhancing mixing, or generally increasing the efficiency of fluid systems (see Gad-el-Hak 1996; Bewley 2001; Kim \& Bewley 2007; Jahanmiri 2010, for reviews on these subjects). For any but the most simple configurations, the governing equations describing the perturbation dynamics commonly require a drastic reduction in the degrees of freedom before low-dimensional compensators can be designed. This model-reduction step inevitably introduces inaccuracies into the design process, which together with typical heuristics about the noise environment (free-stream turbulence, wall roughness, acoustic forcing, etc.) can negatively influence the

$†$ Email address for correspondence: pjschmid@imperial.ac.uk 
performance of controllers, sometimes up to failure in reaching the control objective. Common reduction techniques use Galerkin projections based on proper orthogonal decomposition (POD) modes (Rowley, Colonius \& Murray 2004; Luchtenburg et al. 2006), balanced POD modes (Willcox \& Peraire 2002; Rowley 2005; Ilak \& Rowley 2006) or the eigenvalue realization algorithm (ERA) (Illingworth, Morgans \& Rowley 2012) to arrive at reduced-order models for control applications. The subsequent design of a controller proceeds along standard lines following optimal or robust control theory (see e.g. Burl 1998). The ability of these techniques to produce successful numerical control strategies is well established and documented; an equally successful implementation in an experiment, however, is still lacking, to the best of the authors' knowledge.

System identification, and control design based on the identified model, is an alternative and less explored methodology that extracts an input-output relation between actuator(s) and sensor(s) directly from processing computed or measured data-sequences. Persistent uncertainties and variability in the system, even of unknown origin, are taken into account during the identification process. System identification has been successfully applied, for example, to experimentally suppress cavity tones (Cattafesta et al. 1997; Kegerise, Cabell \& Cattafesta 2004; Cattafesta et al. 2008). Recently, convectively dominated flows have been treated numerically by a combined identification/feed-forward approach in Hervé et al. (2012) and Juillet, Schmid \& Huerre (2013); the investigations of Rathnasingham \& Breuer (1997, 2003) and Lundell (2007) appear to be the only applications of this methodology to a convectively dominated, experimental flow. Of these previous efforts, the work of Rathnasingham \& Breuer (2003) most closely resembles the approach taken in our study, despite their focus on a turbulent boundary layer at a far larger Reynolds number. Even though their type of flow contains a wider range of spatial and temporal scales and a nonlinear dynamics, the control in Rathnasingham \& Breuer (2003) is applied close to the wall in the viscous sublayer $\left(y^{+}<20\right)$ where nonlinear effects are expected to be small or negligible and where feed-forward control can be applied to an essentially linear flow behaviour. In addition, appreciable reduction of disturbance measures could only be accomplished over a streamwise extent of 1.5 local boundary layer thicknesses (measured by $95 \%$ of $U_{\max }$ ), a distance dictated by the separation of upstream and downstream sensors. Our study conceptually builds on the work of Rathnasingham \& Breuer (2003), but explores a no less challenging flow and aims at a more non-local and expansive control of naturally occurring disturbances.

This article presents a feasibility and performance assessment study of a combined system-identification/feed-forward control approach aimed at suppressing naturally occurring perturbations in wall-bounded shear flows under realistic conditions. For simplicity, subcritical (i.e. laminar) channel flow has been chosen as our flow configuration and as a generic representation of more complex wall-bounded, noise-amplifying flows that are dominated by convection.

\section{Experimental set-up and flow characterization}

The experiments for this article were carried out in an open-return tunnel at GALCIT. The tunnel has a total length of $L_{t}=260 \mathrm{~cm}$ and internal cross-sectional dimensions of width $W=15.6 \mathrm{~cm}$ and height $H=2 h=1.2 \mathrm{~cm}$, resulting in a width-to-height ratio $W / H=13$. Air is driven by a simple fan, passes through a small screen (mesh size $1.5 \mathrm{~mm}$ ) and a smooth contracting nozzle of inlet-to-outlet ratio $A=8.3$ and enters the test section of length $L=186 \mathrm{~cm}(L / H=155)$ (see figure 1). 

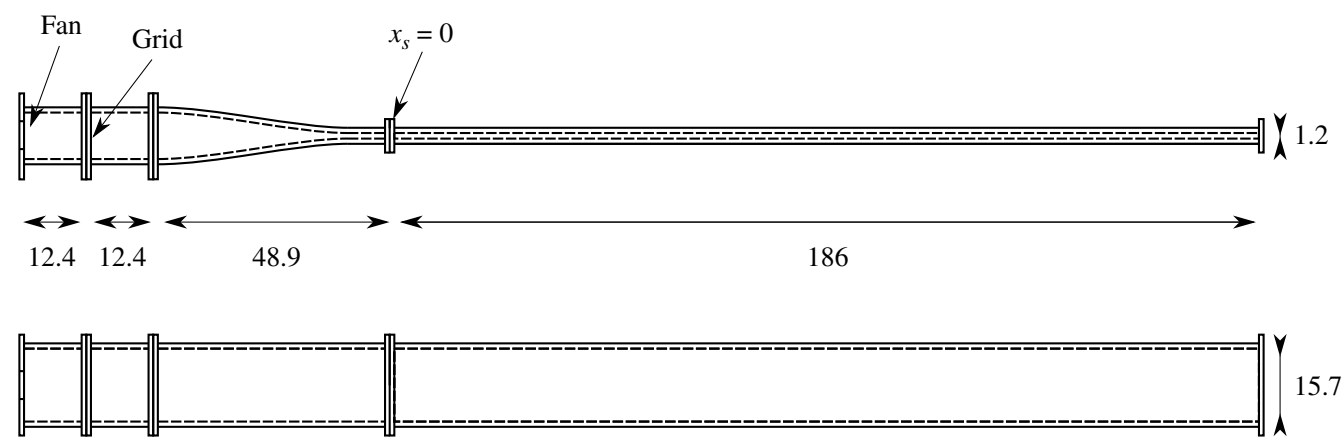

FIGURE 1. Schematic representation of the open-return tunnel used for the experiments (dimensions in $\mathrm{cm}$ ).

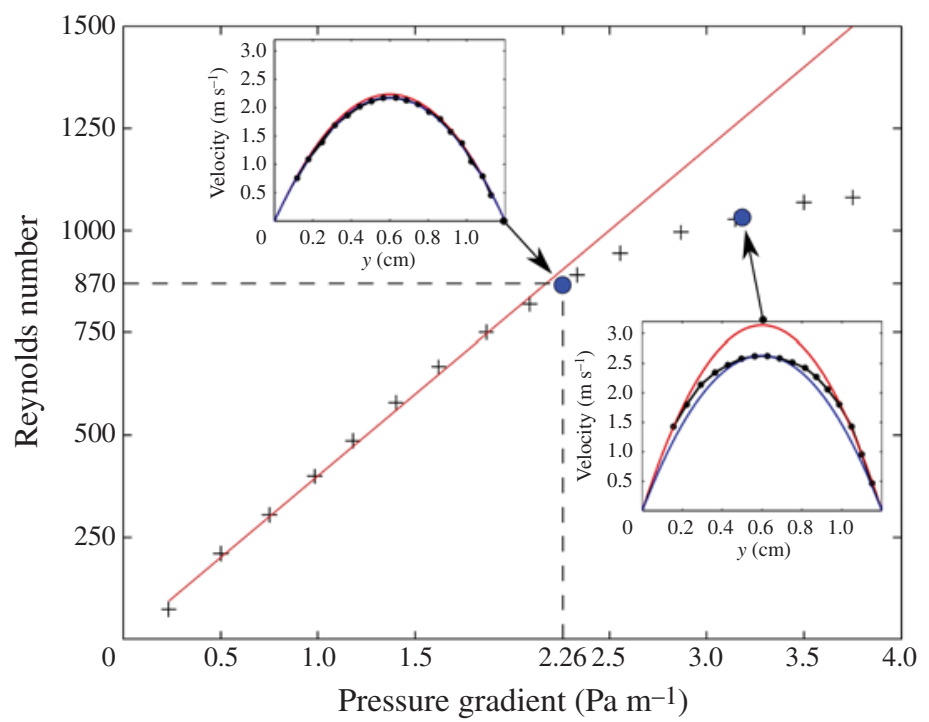

FIgURE 2. (Colour online) Reynolds number as a function of the mean-pressure gradient in the channel. For a Poiseuille flow, the theoretical relation is represented by the straight solid line; the measurements are given by + symbols. The streamwise velocity profile is shown (see insets) for two different Reynolds numbers. At the higher Reynolds number, flattening of the profile in the channel centre can be observed.

The Reynolds number based on the channel half-height $h$ and the maximum speed $U_{\max }$ in the duct is $R e=870$. Measurements of the mean velocity profile as well as the mean-pressure gradient (see figure 2) confirm the Reynolds number as subcritical. At this Reynolds number we still encounter the laminar, parabolic channel-flow profile, while experiencing significant disturbance growth.

Two hot-film sensors (Dantec hot film probe 55R47) were placed on the channel wall at $x_{s}=0$ and $x_{s}=29 \mathrm{~cm}\left(x_{s} / H=24.17\right)$ and operated at constant temperature. The upstream sensor will provide information about the incoming disturbance field, while the downstream sensor will be used to evaluate the control objective. Actuation (blowing/suction) is performed by a computer-controlled $25 \mathrm{cl}$ syringe pump connected to the lower channel wall at $x_{a}=16.5 \mathrm{~cm}\left(x_{a} / H=13.75\right)$ by a small hole of diameter 


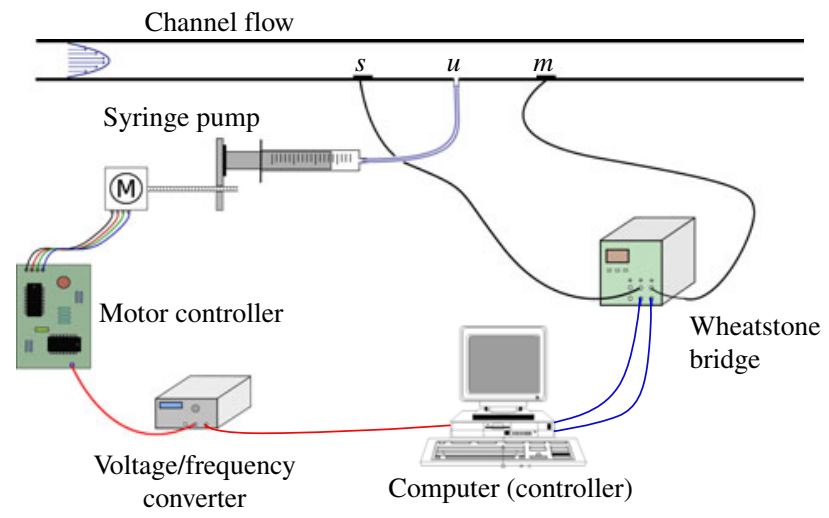

FIGURE 3. (Colour online) Sketch of the full experimental set-up including the air tunnel, all actuation and measurement devices, and data acquisition and processing units.

$0.8 \mathrm{~mm}$. A sketch of the set-up is presented in figure 3. The acquired sensor signals pass via an AN-1005 AA Labs constant-temperature anemometer (CTA) to the computer. The software Labview is used for both the identification and control phase of the study, with a sampling time of $2 \mathrm{~ms}$. The sampling time has been chosen such that the bulk of the disturbance frequency spectrum targeted for control is well below the corresponding Nyquist frequency. Finally, the control signal is processed by a voltage/frequency converter and passed via a motor controller to the stepper unit of the syringe pump. A wide range of choices for actuation is available (see e.g. Gad-el-Hak 1996; Cattafesta et al. 2003; Cattafesta \& Sheplak 2011), including active dimples (Dearing, Lambert \& Morrison 2007) or any active roughness on the wall (Jacobi \& McKeon 2011), plasma actuators (see e.g. Belson et al. 2012), wall oscillations (Quadrio \& Ricco 2004; Moarref \& Jovanovic 2012), synthetic jets (see e.g. Glezer \& Amitay 2002) or general blowing/suction devices (Woodcock, Sader \& Marusic 2012). A syringe pump linked to a stepper motor has been chosen for our study due to its simplicity yet precision in controlling the blowing and suction velocity. Anticipating the application of a linear framework for both system identification and control design, we must ensure that the actuation exercised by the syringe pump can be aptly described by a linear relation. Due to the relatively high Reynolds number, inertia stemming from blowing or suction cannot be neglected in the cross-flow; consequently, the flow response is not symmetric with respect to positive or negative actuation, and linearity cannot be assumed. In fact, the effect of suction on the downstream sensor was nearly negligible when compared to the response to blowing. An improved linear response behaviour can be accomplished by operating the actuator about a small and constant rate of blowing. Small fluctuations about this minor base bleed were then used to suppress the incoming flow perturbations. The constant rate of blowing (of $4.8 \mathrm{~cm}^{3} \mathrm{~s}^{-1}$ which constitutes $0.18 \%$ of the mean volume flux) has been chosen to ensure a positive influx of control fluid from the syringe over the entire control cycle, while keeping its effect on any possible mean-flow modification negligible. In spite of the small mean volume flux of the syringe pump, the induced mean blowing velocity is comparable to the velocity scales in the channel. As a consequence, a mean shear-stress level increase of approximately two standard deviations of the local temporal fluctuations is introduced at the downstream sensor. Nonetheless, experiments verified that this mean component has no noticeable effect 

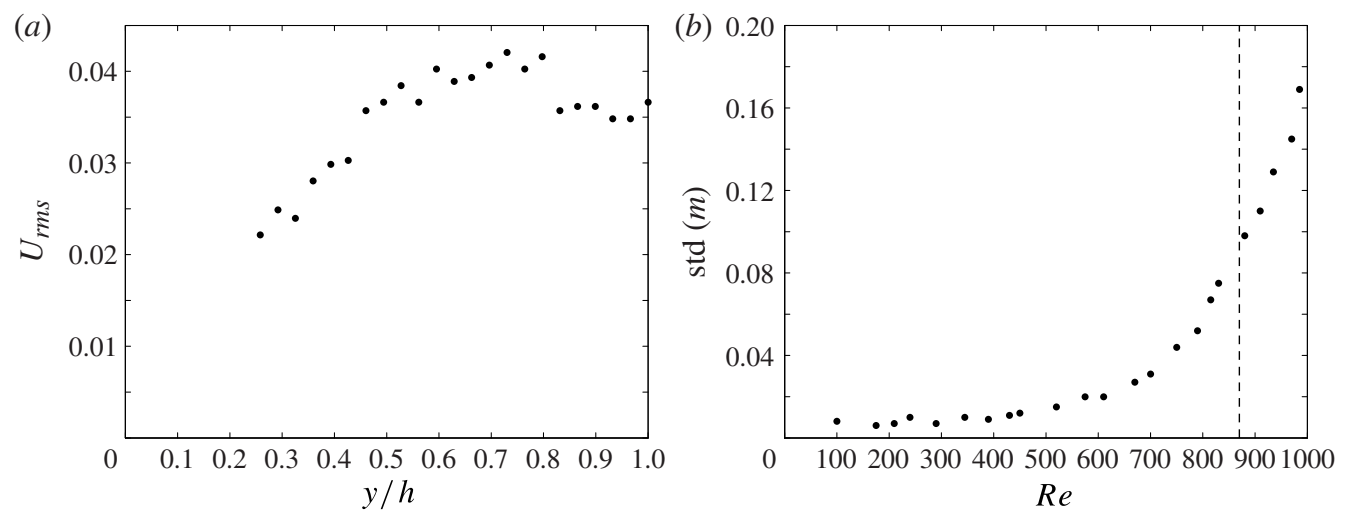

FIGURE 4. (a) Root-mean-square values of the streamwise velocity versus the wall-normal coordinate direction. (b) Standard deviation of the downstream (objective) measurement signal versus the Reynolds number, under off-control conditions.

$\begin{array}{lccccccc}\text { Reynolds number } & 345 & 610 & 700 & 815 & \mathbf{8 8 0} & 935 & 985 \\ \text { Sensor signal standard deviation } & 0.010 & 0.020 & 0.031 & 0.067 & \mathbf{0 . 0 9 8} & 0.129 & 0.169\end{array}$

TABLE 1. Standard deviation of the objective sensor signal as a function of the Reynolds number. The sensor signal fluctuations strongly increase with the Reynolds number. Bold numbers indicate the values for our control results.

on either the system identification step or the ultimate control performance, as only fluctuations are used to identify the transfer functions and only fluctuations are ultimately controlled.

To establish a baseline case, the root-mean-square (r.m.s.) values of the streamwise velocity component have been evaluated and are plotted in figure 4(a). They show a rise from 0.022 to a peak value of 0.042 of the centreline velocity value over a wall-normal extent of 0.258 of the channel half-height to the centreline. In addition, standard-deviation measurements from the downstream (objective) sensor have been taken for the uncontrolled configuration; they are given in figure $4(b)$ displaying a steady rise as the Reynolds number is increased. The Reynolds number for our control results is indicated by the dashed line and yields a standard deviation in the measurement signal of approximately 0.1 . Selective values of these measurements are given in table 1.

\section{System identification and feed-forward control design}

As outlined in the introduction, we will apply a system identification approach to deduce a quantitative model from observed data, which in a second step will be used to design a feed-forward control strategy to minimize the naturally occurring, incoming flow disturbances. The model to be identified assumes a linear relation between the input and output variables. This system identification technique is particularly suited to an experimental setting, since environmental noise and other flow uncertainties are directly reflected in the identified model, without heuristic external user input. For fluid systems that are strongly dominated by convection, 
feedback from downstream sensors is usually futile, while information from upstream becomes crucial (Juillet et al. 2013). The general algorithm and its implementation for convectively dominated fluid problems are presented in Dovetta, Juillet \& Schmid (2014) and Juillet (2013), but a concise description of the essential steps, tailored to our specific configuration, is given in the following sections. Further details on this general approach and on applications to fluid flows can be found in previous work by Cattafesta et al. (2003), Rathnasingham \& Breuer (2003), Hervé et al. (2012) and Juillet et al. (2013).

\subsection{System identification: choice of model structure}

System identification aims at extracting a mathematical model for a dynamical system by observing and processing input-output data only. Procedurally, it involves a least-squares fit of a postulated, general input-output expression to measured input-output sequences. Typical models for system identification include, among others, finite impulse response (FIR) filters, AutoRegressive models with eXogeneous inputs (ARX) or AutoRegressive Moving-Average models with eXogeneous inputs (ARMAX) (see Ljung 1999, for an overview). For our experiment, the simplest structure, an FIR model, has been chosen; more sophisticated models have also been tested, but their added complexity did not yield sufficient improvements to warrant their use. Mathematically, an FIR model of order $\mu_{u}$ takes the form

$$
m(k)=\sum_{j=0}^{\mu_{u}} H_{j}^{u} u(k-j),
$$

where $m(k)$ denotes the output signal of the system at discrete times $t_{k}=k \Delta t$, $u(k-j)$ stands for the input signal of the system at time index $(k-j)$ and $H_{j}^{u}$ is the $j$ th unknown model coefficient (also referred to as the $j$ th Markov parameter). This model expresses the current output measurement as a linear combination of the current and past input measurements. It arises from a classical convolution product, used to describe a causal linear system with input $u$ and output $m$. For our flow configuration, perturbations are predominantly convected downstream which allows the truncation of the infinite convolution product at a finite limit, as shown in (3.1). In this case, the Markov parameters represent the finite discrete impulse response from the actuator $(u)$ to the sensor $(m)$. Information from the upstream sensor $(s)$ also has to be considered. Again, the convective nature of the flow clearly designates this signal as an input to our model, rather than an output of our system, resulting in our final model structure for the experimental input-output dynamics:

$$
m(k)=\sum_{j=0}^{\mu_{u}} H_{j}^{u} u(k-j)+\sum_{j=0}^{\mu_{s}} H_{j}^{s} s(k-j),
$$

where $H_{j}^{s}$ is the $j$ th Markov parameter accounting for the sensor signal $s$. Once the model is chosen, further steps in the system identification consist of adjusting the coefficients $H_{j}^{u}$ and $H_{j}^{s}$ to optimally fit the measured data.

\subsection{System identification: least-squares minimization}

Once input and output data sequences have been recorded, the best-fit Markov parameters can be determined by minimizing (in a least-squares sense) the difference 
between the measured signal $m$ and the estimated output from the model given $u$ and $s$. In mathematical terms, we solve the optimization problem

$$
\left(H_{j}^{u}, H_{j}^{s}\right)_{j=1, \mu_{u / s}}=\operatorname{argmin} \sum_{k=0}^{N}\left\|m(k)-\sum_{j=0}^{\mu_{u}} H_{j}^{u} u(k-j)-\sum_{j=0}^{\mu_{s}} H_{j}^{s} s(k-j)\right\|^{2}
$$

for data sequences $\{u, s, m\}$ of length $N+1$. The above expression can be recast in matrix form as

$$
\boldsymbol{H}=\operatorname{argmin}\|M-\boldsymbol{H} \boldsymbol{\Phi}\|_{2}^{2}
$$

with $M=(m(0), \ldots, m(N))$ and $\boldsymbol{H}=\left(H_{0}^{u}, \ldots, H_{\mu_{u}}^{u}, H_{0}^{s}, \ldots, H_{\mu_{s}}^{s}\right)$. The matrix $\boldsymbol{\Phi}$ contains the input data in the form

$$
\boldsymbol{\Phi}=\left(\begin{array}{cccc}
u(0) & u(1) & \cdots & u(N) \\
u(-1) & u(0) & \cdots & u(N-1) \\
\vdots & \vdots & & \vdots \\
u\left(-\mu_{u}\right) & u\left(1-\mu_{u}\right) & \cdots & u\left(N-\mu_{u}\right) \\
\hline s(0) & s(1) & \cdots & s(N) \\
s(-1) & s(0) & \cdots & s(N-1) \\
\vdots & \vdots & & \vdots \\
s\left(-\mu_{s}\right) & s\left(1-\mu_{s}\right) & \cdots & s\left(N-\mu_{s}\right)
\end{array}\right)
$$

The optimization problem (3.4) can then be solved for the set of Markov parameters $\boldsymbol{H}$ as follows:

$$
\boldsymbol{H}=M \boldsymbol{\Phi}^{\dagger},
$$

where $\boldsymbol{\Phi}^{\dagger} \equiv \lim _{\epsilon \rightarrow 0} \boldsymbol{\Phi}^{\mathrm{T}}\left(\boldsymbol{\Phi} \boldsymbol{\Phi}^{\mathrm{T}}+\epsilon \boldsymbol{I}\right)^{-1}$ indicates the Moore-Penrose pseudo-inverse of $\boldsymbol{\Phi}$ with $\epsilon$ as a regularization parameter (Penrose 1955; Tikhonov 1963). Alternatively, the Markov parameters can be determined sequentially from an unforced $(u=0)$ experiment followed by a forced $(u \neq 0)$ experiment.

\subsection{Feed-forward control design}

The final step consists of the design of a control law from the identified Markov parameters. For simplicity, we assume that the two sets of Markov parameters (impulse responses) have identical lengths, $\mu_{s}=\mu_{u} \equiv \mu$. In practice, this choice is not detrimental as long as $\mu$ is sufficiently large. Within a feed-forward setting, the final control law is in the form of a direct causal relation between the upstream sensor signal $s$ and the actuator signal $u$, which can be expressed, analogously to the analysis above, as an impulse response from $s$ to $u$ given by a set of unknown coefficients $L \equiv\left(L_{0}, \ldots, L_{\mu}\right)$. Causality requires $L_{k}=0$ for $k<0$. Figure 5 gives a graphical interpretation of this issue. The coefficients $L_{k}$ can be readily determined by assuming a discrete impulse in $s$ (that is, $s(0)=1$ and $s(k)=0$ for $k>0$ ), for which the response in $m$ is given according to (3.2) by

$$
m(k)=\sum_{j=0}^{\min (\mu, k)} H_{j}^{u} L_{k-j}+H_{k}^{s} .
$$




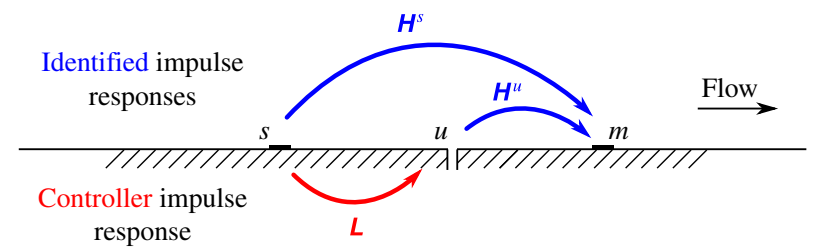

FIGURE 5. (Colour online) Identified impulse responses from the spy sensor $s$ to the objective sensor $m$ and from the actuator $u$ to objective sensor $m$. The controller impulse response $L$ is also represented.

From this expression, the coefficients $L_{k}$ can be computed by rendering the output $m(k)$ minimal for any $k$. This latter optimization problem is equivalent to minimizing the cost functional $\boldsymbol{J}(\boldsymbol{L})$ given in matrix form as

$$
\boldsymbol{J}(\boldsymbol{L})=\left\|\boldsymbol{L} \boldsymbol{\Psi}+\boldsymbol{H}^{\boldsymbol{s}}\right\|_{2}^{2}+\alpha\|\boldsymbol{L}\|_{2}^{2},
$$

with $\boldsymbol{H}^{s}=\left(H_{0}^{s}, \ldots, H_{\mu}^{s}\right)$ and

$$
\boldsymbol{\Psi}=\left(\begin{array}{cccc}
H_{0}^{u} & H_{1}^{u} & \cdots & H_{\mu}^{u} \\
0 & H_{0}^{u} & \cdots & H_{\mu-1}^{u} \\
\vdots & \vdots & \ddots & \vdots \\
0 & 0 & \cdots & H_{0}^{u}
\end{array}\right)
$$

The second term in (3.8) represents a penalization of the actuator signal with $\alpha$ as a penalty cost parameter. Physically, this term limits the amplitude of the actuator signal; mathematically, it acts as a (Tikhonov) regularization of the problem. The solution of the minimization problem (3.8) leads to the controller impulse response

$$
\boldsymbol{L}=\boldsymbol{H}^{\boldsymbol{s}} \boldsymbol{\Psi}^{\mathrm{T}}\left(\boldsymbol{\Psi} \boldsymbol{\Psi}^{\mathrm{T}}+\alpha \boldsymbol{l}\right)^{-1} .
$$

The controller impulse response $\boldsymbol{L}$ given in (3.10) fully describes the controller dynamics; this concludes the control design procedure.

\section{Results}

For the identification phase of the proposed FIR model a choice has to be made as to the actuator excitation. Stochastic signals (e.g. white noise) and deterministic forcings (e.g. chirp) have to be weighed against theoretical advantages for the identification and experimental feasibility. A good compromise is given by a random binary signal: it excites a sufficiently wide frequency range, and its standard deviation equals its maximum value, a property (known as a unit crest factor) which proves advantageous for system identification (see Ljung 1999). A typical actuator signal during the identification phase is shown in figure 6, alternating between constant excitation and rest. A superiority of random over deterministic signals has also been observed in numerical experiments (see, e.g. Juillet et al. 2013).

Two impulse responses (Markov parameter sequences) result from processing the input/output data sequences according to the system identification algorithm outlined above; they are presented in figure 7. A typical delay of $0.2 \mathrm{~s}$ can be observed for the impulse response from the upstream to the downstream sensor (figure $7 a$ ) which 


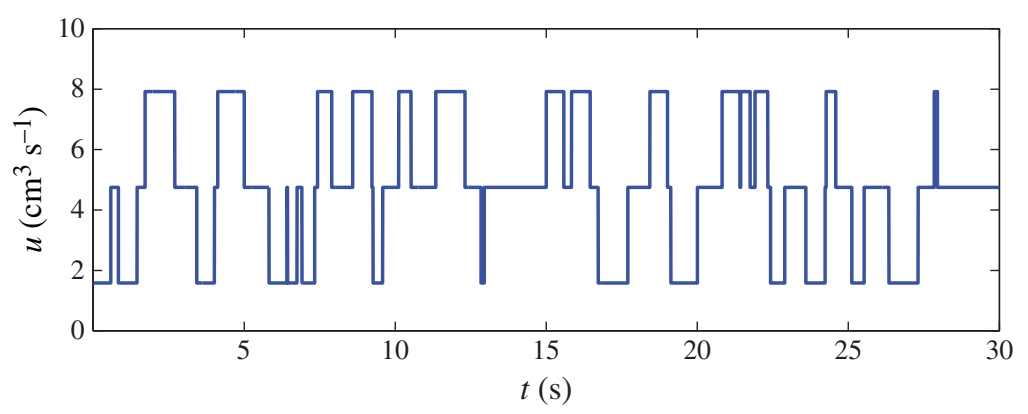

FIGURE 6. (Colour online) Representative sample of a random binary actuator signal used during the system identification phase. Notice the mean base bleed of $4.8 \mathrm{~cm}^{3} \mathrm{~s}^{-1}$.

(a)

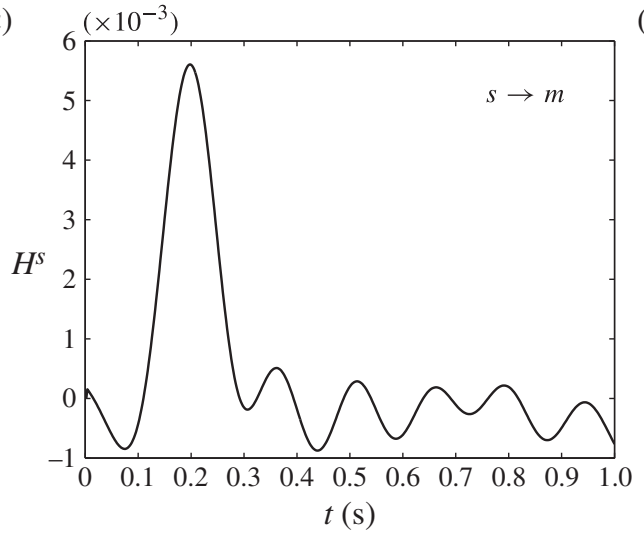

(b)

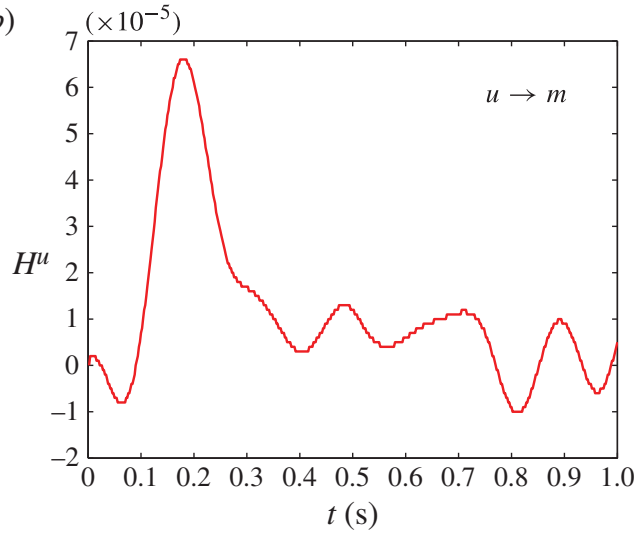

FIgURE 7. (Colour online) Identified impulse response (a) from the upstream $s$ to the downstream sensor $m,(b)$ from the actuator $u$ to the downstream sensor $m$. The model order $\mu$ has been adjusted until independence from this parameter has been reached.

translates into a perturbation group velocity of $U_{g}=1.46 \mathrm{~m} \mathrm{~s}^{-1}=0.68 U_{\max }$. Nearly the same characteristic time of $0.2 \mathrm{~s}$ is also measured for the impulse response from the actuator to the downstream (objective) sensor (figure 7b). This finding may, at first sight, seem surprising given the fact that the actuator is placed much closer to the downstream sensor. However, one has to keep in mind that the identification procedure describes the system from the computer-generated input signal to the output signal recorded by the same computer, mathematically represented by the Markov parameter sequence $H_{j}^{u}$. Between these two signals lies a multi-component system (see figure 3) whose elements add delays to the overall response time. Based on the already identified group velocity $U_{g}$, the convective time from actuator to downstream sensor is estimated as $0.09 \mathrm{~s}$, which leaves a delay of $0.1 \mathrm{~s}$ stemming from the electrical and mechanical devices. This matter also illustrates the strength of system identification in accommodating typical imperfections and shortcomings in the system; an approach based on common reduced-order models could not manage this type of challenge.

In order to validate the identified model, the downstream sensor signal $m$ used for the identification is compared with the predicted output from (3.2). Figure 8 shows a pleasing agreement between the predicted (black) and measured (grey) signal. In 


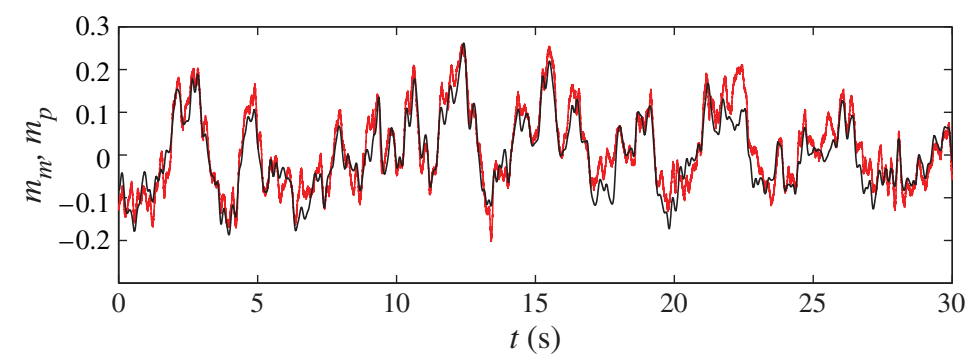

FIGURE 8. (Colour online) Comparison of the measured downstream sensor signal $m_{m}$ (grey) and the predicted value $m_{p}$ (black) from the identified model. A prediction accuracy of $60 \%$ of the measured signal is obtained.

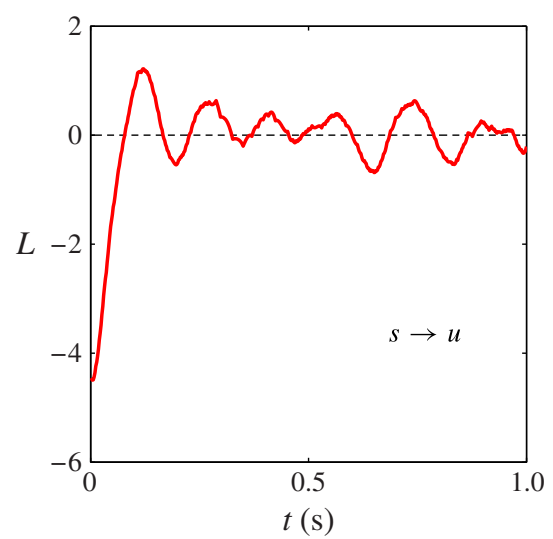

FIGURE 9. (Colour online) Controller impulse response which describes the control signal triggered by an impulse in the upstream sensor $s$.

quantitative terms, a better than $60 \%$ match has been achieved corresponding to a relative error, measured as the standard deviation of the absolute signal difference to the standard deviation of the uncontrolled signal, of less than $40 \%$. More importantly, the measured and estimated signals are closely locked in phase which instills confidence in the design and implementation of a controller based on this model.

Application of the control design (3.10) with the penalization parameter of $\alpha=10^{-8}$ results in the controller impulse response presented in figure 9. A strong negative peak at $t=0$ is observed, reminiscent of opposition control; the entire response, however, is more complex and therefore more effective than classical opposition control strategies (see e.g. Rebbeck \& Choi 2006). The controller seems to react immediately after detection of an incoming perturbation in $s$. This is to be expected, since the two identified impulse responses showed peaks at nearly the same time (recall figure 7). The lack of delay, despite the physical separation of the upstream sensor and actuator, can be attributed to the cumulative reactive gap in the syringe pump system. This gap has been accounted for during the system identification step and results in an immediate action of the controller, once a signal in $s$ has been detected. Moving the sensor $s$ further upstream may introduce a more pronounced delay, while the accuracy of the system identification will degrade; a compromise between identification accuracy and control response has to be reached. 
(a)

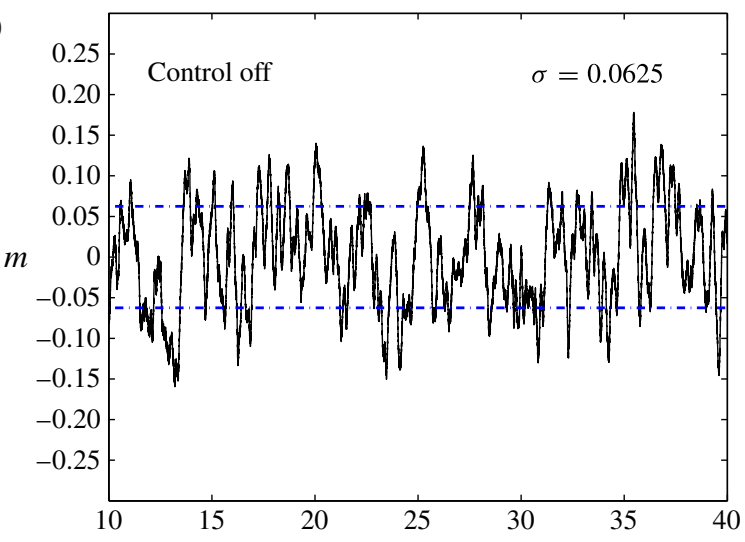

(b)

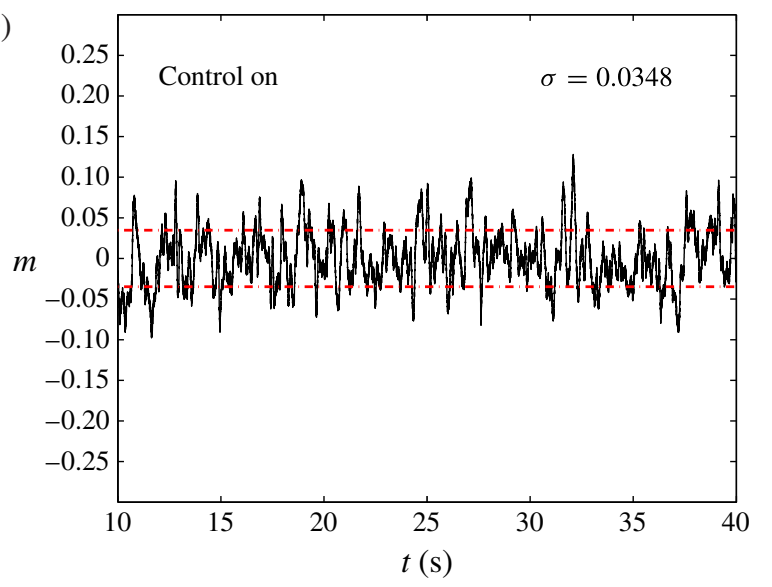

FIgURE 10. (Colour online) Objective sensor signal $m$ measured $(a)$ with the control switched off, $(b)$ with the control activated. A reduction of $44 \%$ in the signal's standard deviation has been accomplished.

The controller represented by its impulse response (figure 9) has been implemented in our experiment. Representative results of the controlled and uncontrolled flow are shown in figure 10: the downstream sensor signal is recorded for a period of $30 \mathrm{~s}$ with the control off (figure 10a) or on (figure 10b). For this specific realization the uncontrolled signal has a standard deviation of $\sigma=0.0625$ which can be reduced by $44 \%$, to a value of $\sigma=0.0348$, once the control has been activated. Statistics on the control efficiency have been gathered by repeating the control experiment more than 25 times (for a total recorded time of more than $13 \mathrm{~min}$ ). On average, a robust reduction in standard deviation by $45 \%$ has been measured.

Strictly speaking, only a reduction in the downstream (objective) sensor signal can be expected, owing to the formulation of the control objective in (3.8); further reductions downstream of the objective sensor are neither enforced nor guaranteed. Nonetheless, we are interested in assessing the streamwise extent of the controlled flow downstream. To this end, we place an additional, third sensor at $x_{s 2}=14 \mathrm{~cm}\left(x_{s 2} / H=11.67\right)$ farther downstream from the objective sensor. With the feed-forward controller switched on, this sensor still registers an average reduction in signal standard deviation of $30 \%$ compared to the uncontrolled signal. This result 


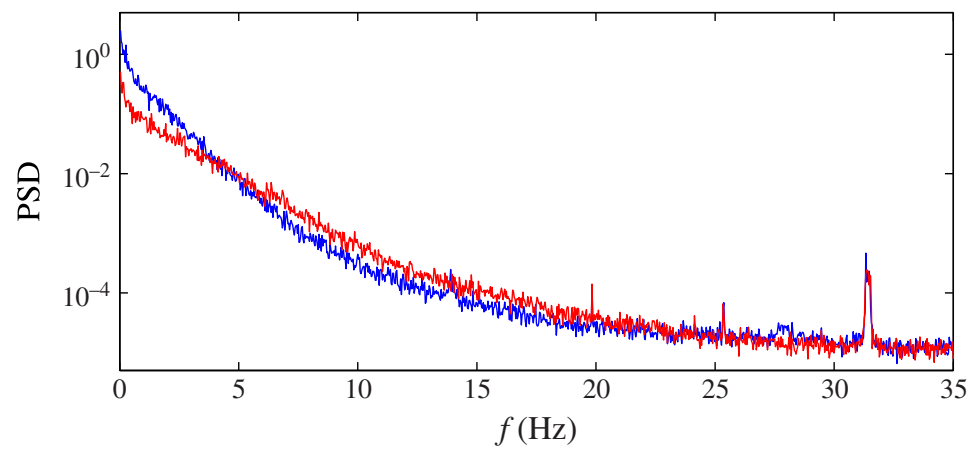

FIGURE 11. (Colour online) Power spectral density of the downstream (objective) sensor signal (grey) without control and (black) with control.

illustrates the effectiveness of the feed-forward controller, even beyond the strict limits of its design.

Finally, the power spectral densities of the objective sensor signals with and without control are displayed in figure 11. The dynamics in the channel are clearly dominated by low frequencies, and the controller has been able to reduce the low-frequency spectral density by nearly a factor of four. Frequencies in the range of $4.5-25 \mathrm{~Hz}$ are amplified by the controller; however, they represent a very small fraction of the entire energy content, such that a significant net reduction in energy prevails. Small peaks at frequencies above $20 \mathrm{~Hz}$ are still 1000 times smaller than the spectral density in the lowest frequencies; their origin can be ascribed to mechanical or electro-mechanical oscillations in the channel. In short, the uncontrolled disturbance spectrum, dominated by low frequencies, could be successfully reduced by our syringe-pump control device, at the expense of a small and irrelevant increase in the higher frequencies.

\section{Summary and conclusions}

System identification based on an FIR model and followed by a feed-forward control design has been applied to an experiment on subcritical channel flow $(R e=$ 870 ) to decrease the naturally occurring perturbation magnitude. A blowing/suction strategy using a simple syringe pump was capable of reducing the standard deviation of the downstream sensor signal on average by $45 \%$; a second sensor, placed a posteriori farther downstream, could still record a $30 \%$ reduction in standard deviation, which suggests a decrease in disturbance level beyond the localized region for which the control strategy has been designed.

The significance of this reduction (and the present study) must be contrasted with results previously obtained using similar techniques. Rathnasingham \& Breuer (2003) applied system identification and feed-forward techniques to a turbulent boundary layer at $\operatorname{Re}_{\delta^{*}}=1960$, nearly 20 times the Reynolds number considered here. Their control efforts have, however, been concentrated in the near-wall region at values of $y^{+}<20$, where the flow behaves sufficiently linearly to justify a linear identification and control approach. Effectiveness of a control effort must be measured not only by the percentage of reduction in the measurement signal, but also by the spatial extent of a significant reduction. By simply placing the objective sensor in the vicinity of the upstream sensor, a straightforward identification and a seemingly effective control can be forced. Any reduction thus accomplished, however, would be rather localized 
in the neighbourhood of the sensors. The upstream-to-downstream sensor distance in Rathnasingham \& Breuer (2003) was $3.3 \mathrm{~cm}$ or $1.5 \delta$ based on the $95 \%$ boundary layer thickness. In contrast, the equivalent sensor distance is $63 \delta$ in our study and is hence less localized, as demonstrated above by evaluating the signal of an additional sensor which has not been accounted for in the identification and control design process. While control of disturbances in turbulent boundary layers over large spatial extents certainly is very difficult to achieve, the results presented here point towards key differences between the two configurations which may generate interest in the present study.

The level of reduction presented here is less than what can be accomplished in numerical simulations with similar techniques (which can be attributed to the uncompromised conditions and idealizations of the computations, especially regarding noise sources and actuator modelling). Nonetheless, it exceeds previous values from experimental studies by a considerable margin: as mentioned above, Rathnasingham \& Breuer (2003) apply related techniques to control natural flow disturbances in a turbulent boundary layer at $R e=1960$ (based on the momentum thickness) and report a maximum localized reduction of $30 \%$ in the streamwise velocity fluctuations; more recently, Lundell (2007) used a threshold-and-delay control algorithm and achieved a maximum disturbance reduction of $18 \%$. A related study by Jacobson \& Reynolds (1998) attempted to control disturbances in an $R e=600$ boundary layer, but introduced the upstream perturbations artificially and deterministically.

The technique introduced in this article is capable of identifying and controlling a naturally occurring disturbance environment in an effective, efficient and robust manner. It provides a promising direction in the further pursuit of control of wall-bounded shear flows dominated by convective processes.

\section{Acknowledgement}

The authors wish to thank K. Rosenberg for his assistance in obtaining the measurements of the base-flow characteristics.

\section{REFERENCES}

Belson, B. A., Hanson, R. E., Palmeiro, D., Lavoie, P., Meidell, K. \& Rowley, C. W. 2012 Comparison of plasma actuators in simulations and experiments for control of bypass transition. AIAA Paper 2012-1141.

Bewley, T. R. 2001 Flow control: new challenges for a new renaissance. Prog. Aerosp. Sci. 37 (1), 21-58.

BURL, J. 1998 Linear Optimal Control. Prentice Hall.

Cattafesta, L. N., Garg, S., Choudhari, M. \& Li, F. 1997 Active control of flow-induced cavity resonance. AIAA.

Cattafesta, L. N. \& Sheplak, M. 2011 Actuators for active flow control. Annu. Rev. Fluid Mech. 43, 247-272.

Cattafesta, L. N., Song, Q., Williams, D. R., Rowley, C. W. \& Alvi, F. S. 2008 Active control of flow-induced cavity oscillations. Prog. Aerosp. Sci. 44, 479-502.

Cattafesta, L. N., Williams, D., Rowley, C. W. \& Alvi, F. 2003 Review of active control of flow-induced cavity resonance. AIAA Paper 2003-3567.

Dearing, S., Lambert, S. \& Morrison, J. 2007 Flow control with active dimples. Aeronaut. J. $705-714$.

Dovetta, N., Juillet, F. \& Schmid, P. J. 2014 Data-based model-predictive control design for convectively unstable flows. Phys. Fluids (submitted). 
Gad-El-HaK, M. 1996 Modern developments in flow control. Appl. Mech. Rev. 49 (7), 365-379. Glezer, A. \& Amitay, M. 2002 Synthetic jets. Annu. Rev. Fluid Mech. 34, 503-529.

Hervé, A., Sipp, D., Schmid, P. J. \& SAmuelides, M. 2012 A physics-based approach to flow control using system identification. J. Fluid Mech. 702, 26-58.

IlaK, M. \& Rowley, C. W. 2006 Reduced-order modeling of channel flow using traveling POD and balanced POD. AIAA Paper 2006-3194.

Illingworth, S. J., Morgans, A. S. \& Rowley, C. W. 2012 Feedback control of cavity flow oscillations using simple linear models. J. Fluid Mech. 709, 223-248.

JACOBI, I. \& MCKeON, B. J. 2011 Dynamic roughness perturbation of a turbulent boundary layer. J. Fluid Mech. 688, 258-296.

Jacobson, S. A. \& ReYnolds, W. C. 1998 Active control of streamwise vortices and streaks in boundary layers. J. Fluid Mech. 360, 179-211.

JAHANMiRI, M. 2010 Active flow control: a review. Tech Rep. 2010:12. Chalmers University of Technology.

JUILlet, F. 2013 Control of amplifier flows using subspace identification techniques. PhD thesis, Ecole Polytechnique.

Juillet, F., Schmid, P. J. \& Huerre, P. 2013 Control of amplifier flows using subspace identification techniques. J. Fluid Mech. 725, 522-565.

Kegerise, M., Cabell, O. H. \& Cattafesta, L. N. 2004 Real-time adaptive control of flowinduced cavity tones. AIAA Paper 2004-0572.

Kim, J. \& Bewley, T. R. 2007 A linear systems approach to flow control. Annu. Rev. Fluid Mech. 39 (1), 383-417.

LJUNG, L. 1999 System Identification, Theory for the User, 2nd edn. Prentice Hall PTR.

Luchtenburg, D. M., Tadmor, G., Lehmann, O., Noack, B. R., King, R. \& Morzyński, M. 2006 Tuned POD Galerkin models for transient feedback regulation of the cylinder wake. AIAA-Paper 2006-1407.

LUNDELL, F. 2007 Reactive control of transition induced by free-stream turbulence: an experimental demonstration. J. Fluid Mech. 585, 41-71.

Moarref, R. \& Jovanovic, M. R. 2012 Model-based design of transverse wall oscillations for turbulent drag reduction. J. Fluid Mech. 707, 205-240.

Penrose, R. 1955 A generalized inverse for matrices. Proc. Camb. Phil. Soc. 51, 406-413.

QUADRIO, M. \& RicCO, P. 2004 Critical assessment of turbulent drag reduction through spanwise wall oscillations. J. Fluid Mech. 521, 251-271.

Rathnasingham, R. \& Breuer, K. S. 1997 System identification and control of turbulent flows. Phys. Fluids 9, 1867-1869.

Rathnasingham, R. \& Breuer, K. S. 2003 Active control of turbulent boundary layers. J. Fluid Mech. 495, 209-233.

RebBeck, H. \& CHOI, K. S. 2006 A wind-tunnel experiment on real-time opposition control of turbulence. Phys. Fluids 18, 035103.

Rowley, C. W. 2005 Model reduction for fluids using balanced proper orthogonal decomposition. Intl J. Bifurcation Chaos 15 (3), 997-1013.

Drowley, C. W., Colonius, T. \& Murray, R. M. 2004 Model reduction for compressible flows using POD and Galerkin projection. Physica 189, 115-129.

TikHonov, A. N. 1963 Solution of incorrectly formulated problems and the regularization method. Dokl. Akad. Nauk USSR 151, 501-504.

Willcox, K. \& Peraire, J. 2002 Balanced model reduction via the proper othogonal decomposition. AIAA J. 40 (11), 2323-2330.

Woodcock, J. D., SAder, J. E. \& Marusic, I. 2012 Induced flow due to blowing and suction flow control: an analysis of transpiration. J. Fluid Mech. 690, 366-398. 